\title{
THE MENOPAUSE
}

\author{
By G. I. M. SwYer, D.M., D.Phil., M.R.C.P. \\ Consultant Endocrinologist, Department of Obstetrics and Gynaecology, University College Hospital, London, W.C.I
}

The reproductive period in women is commonly very much shorter than the total life span. The main reason for this is the continual loss or degeneration of potential ova, and the absence of any provision for their replacement. When no more ova remain, reproductive ability obviously comes to an end. On the other hand, the endocrine activity of the ovary does not terminate in this abrupt manner, and so there is a phase of waning function. This phase begins before the menstrual periods cease and continues for some time afterwards. The whole phase is properly called the climacteric; but the term is sometimes confined to the interval between the beginning of ovarian failure and the menopause, or cessation ot the menstrual periods.

Physiology of the Climacteric. In contrast to spermatogenesis, which is essentially a function of sexually mature males, oögenesis-the actual production of oöcytes-is probably purely a foetal activity. Though the more or less classical view, as put forward by Swezy and Evans (1930), was that the production of new oöcytes from the germinal epithelium continued throughout adult life, the idea was opposed by Simkins (1932), and the accurate observations of Mandl and Zuckerman (I95I, a, b, c), and Mandl, Zuckerman and Patterson (1952) have failed to substantiate it. Of the 400,000 (more or less) primary oöcytes present in the two ovaries at birth, only a small proportion, perhaps 400 , are destined to take part in ovulation. The remainder disappear through the process ot atresia. Loss of oöcytes by atresia begins at least as early as birth and is indeed most active berore puberty, for the ovary of the new-born has many thousands more primary follicles than that of the adolescent girl. During the proliferative phase of each menstrual cycle, several follicles commence to grow but only one reaches the stage of ovulation. The remainder, outstripped by the 'chosen' follicle, regress and become atretic. In this way, during each cycle some 30 or 40 follicles are lost by atresia for each one by ovulation. Since the ovarian hormones are secreted by the follicles or their derivatives, it is clear that when few or no follicles remain, ovarian hormone production must fall to a low level or cease altogether.

The age at which the climaicteric commences varies in different women in much the same way as does that at which the menarche occurs; it is in fact more or less normally distributed. Various factors-racial, hereditary (other than racial), general health, sociological and so on-no doubt do determine it, and diseases of various kinds directly affecting ovarian physiology can accelerate it.

It has often been supposed that there is a relationship between the age of the menarche and that of the menopause; it has been suggested that the earlier the occurrence of the former, the later is that of the latter. This is probably not true: in an investigation of the menopause of 1,000 women (Lancet, 1933), it was found that the average age at the menopause for women whose menarche occurred at 13 years was 47.3 years; while that for women whose menarche occurred at 18 years was 47.5 years. Occasionally, the menopause occurs at a very early age-even before 20 years; in these cases there is nearly always an underlying endocrine abnormality. There have also been reports of the continuation of menstruation until very advanced years - up to the age of I04 in one instance (Novak, I92I). In general, however, prolongation of the menopause after the age of 55 calls for gynaecological examination to exclude the possibility of genital malignancy. There is good reason to suppose that many of the patients with delayed menopause reported in ancient literature had oestrogen-producing tumours of the ovary (such as granulosa cell tumours). It is, or course, equally important, or even more so, to make a gynaecological examination if, after the menopause has definitely occurred, genital bleeding should reappear. Although this is frequently of benign cause (particularly if injudicious oestrogen treatment for menopausal symptoms has been given) all too commonly malignant disease of the cervix or body of the uterus is found to be responsible.

When the number of ovarian follicles has 


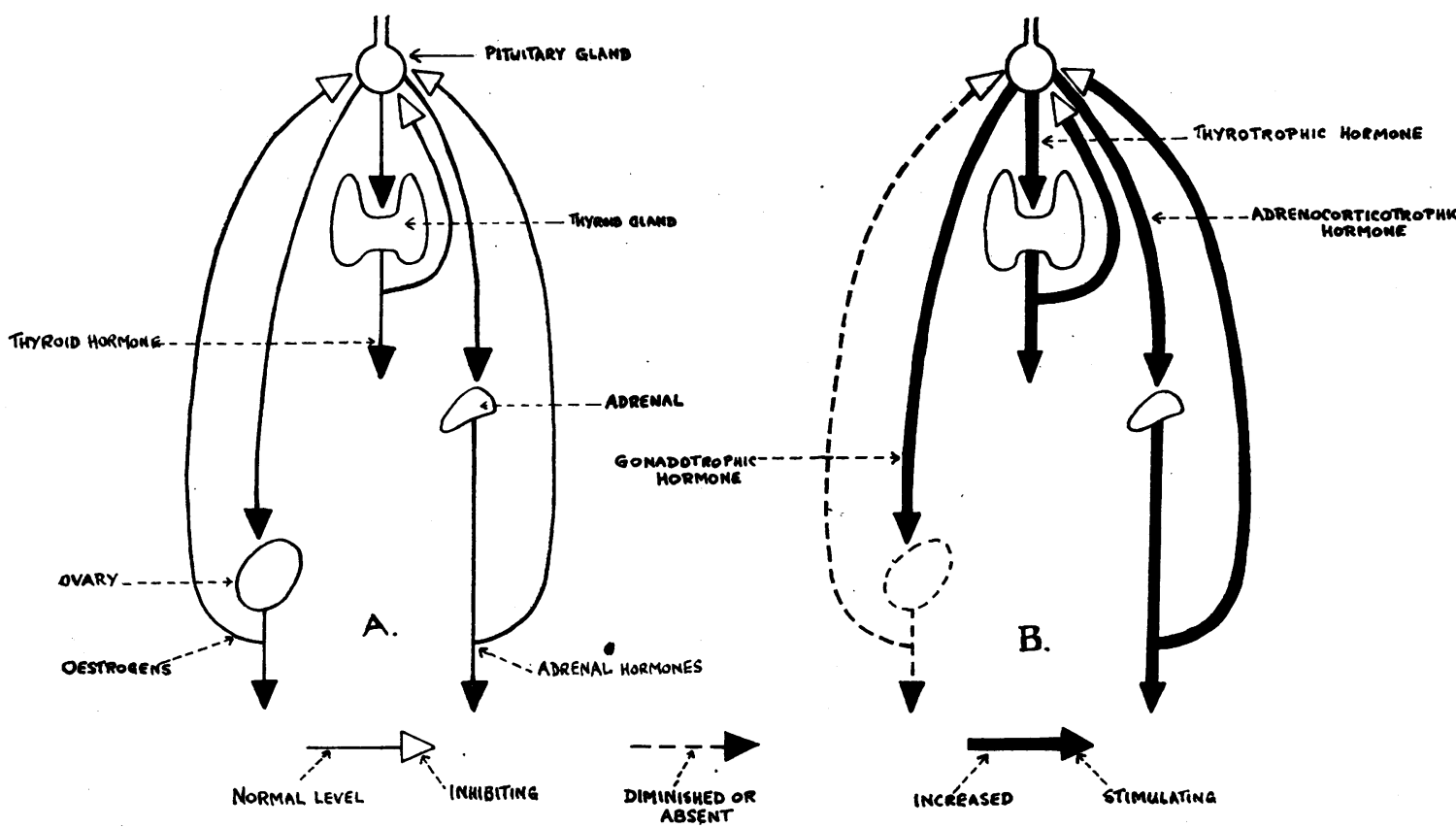

A. Normal hormonal relationships between the anterior lobe of the pituitary gland and the ovaries, thyroid and adrenal glands. B. With failure of oestrogen production by the ovaries at the climacteric, the pituitary becomes hyperactive and so the thyroid and adrenals produce increased amounts of their secretions.

become significantly reduced, although more or less regular cyclic activity may continue for some time, ovulation ceases to occur in every cycle. Later, ovulation ceases altogether and by this time some irregularity of the cycles has usually become apparent. Anovular cycles may continue for some time, the bleeding becoming more infrequent and scantier, eventually to cease altogether. In other women, the menopause may take the form of an abrupt cessation of previously regular periods. Yet a further variant is that in which the alterations in ovarian hormone production lead to the development of irregular, prolonged and often heavy bleeding-climacteric menorrhagia.

The gradual lessening of ovarian endocrine activity during the climacteric leads to many secondary changes. Foremost among these are regression of the genital organs-uterus, vagina, vulva and breasts. But in many women the changes are almost imperceptibly slow and long after the menopause, little definite evidence of genital regression may be seen. In particular, the vaginal smear in post-menopausal women often reveals little evidence of significant oestrogenic deficiency. However, atrophy of the vaginal epithelium may be the cause of post-menopausal bleeding. The tendency to gain weight, which is commonly seen at this stage of life, leading to so-called ' middle-age spread' has been thought to result from failing ovarian function, but this not likely. A more probable explanation is that as part of the general ageing process, less energy is expended; but, because the appetite remains unchanged, a previous balance between energy intake (in the food) and that expended in the day's activities becomes converted into an excess of intake, thereby leading to deposition of fat. It is said that this fat has a peculiar distribution, accumulating mainly around the abdomen, hips and thighs. But perhaps it is distributed in the same way as it would have been, given a comparable weight gain, in earlier years. One possible explanation for the alleged special distribution is that the normal ageing process is accompanied by a relatively greater loss of adipose tissue cells in other parts of the body and that, $\frac{}{0}$ when new fat is laid down, most appears where the adipose cells remain in greater abundance.

Further secondary effects of ovarian failure are $N$ changes in the activity of other endocrine glands (Fig. I). These are most important for a.complete $\omega$ understanding of the physiology of the climacteric. Because of the progressive failure of ovarian response to pituitary gonadotrophic stimulation, the modifying action of the ovarian hormones on the activity of the pituitary gland becomes less and less. With the reduced inhibitory effect of oestrogen, the production of pituitary gonado- $\frac{\stackrel{?}{\oplus}}{\mathrm{Q}}$ 
trophin (mostly of follicle stimulating type) increases, so that in post-menopausal women a great excess can be detected in the urine. Sometimes this gives rise to false pregnancy-diagnosis tests, thus confusing an already delicate issue if the women fears (or hopes) that the delayed period caused by the climacteric is due to pregnancy. Along with this increase in pituitary gonadotrophin production, there is probably also an increase in the output of thyrotrophic and adrenocorticotrophic hormones. These find respossize target organs, and so hyperactivity of the thyroid and adrenal cortex may ensue. It is probably the combination of falling oestrogen level and increased output of thyroid and adrenal hormones which is responsible for most of the untoward effects which may be experienced by women at this stage. An alternative view has supposed that the pituitary over-activity itself is responsible for the climacteric symptoms; but since these are often suppressed by very small doses of oestrogen which have no detectable effect on the pituitary hyperactivity, it seems far more probable that the falling oestrogen level is the more important factor.

The increased activity of the adrenal cortex tends to restore the endocrine balance by taking over some of the functions of the ovary. Thus, there is little doubt that most of the oestrogen which circulates in post-menopausal women, when the ovaries have become quite functionless, is secreted by the adrenal cortex. This oestrogen, and the other adrenal cortical hormones produced in increased amounts, serve to depress the excessive pituitary activity and so reduce the thyroid over-activity. The tendency to develop a considerable growth of hair on the face seen in some women after the menopause is probably directly due to the increased output of androgens by the hyperactive adrenal. With still further advance in age, it is likely that some degree of refractoriness occurs in both the adrenal and the thyroid gland, so that in these older women the clinical picture may suggest some deficiency of function of both these glands.

The Climacteric Syndrome. The precise frequency with which climacteric symptoms of more than minor degree are experienced is uncertain. Hamblen (1947) quotes one authority who estimated 75 per cent. of all women as suffering from distressing symptoms at the climacteric, and other authorities who held that 70 to 90 per cent. of climacteric woman experience no symptoms materially interfering with general health, or with domestic or social activities.

The symptoms of the climacteric syndrome consist mainly of psychological disturbances and vasomotor instability. There is no doubt that the former element is the more important, for it is just those women who have, or through force of circumstances develop, psychological inadequacy, who are most liable to suffer from climacteric symptoms and who do so most severely. Two classes of women provide the majority of the climacteric sufferers. At one end of the social scale is the woman of wealth and social standing, for whom the menopause is a reminder which cannot be ignored of advancing years with their attendant loss of good looks, sex appeal and consequent dominance in her social circle. At the other end is the woman who has led a frustrated existence, deprived of good looks and the activities open to the more fortunate, who has been unwanted and unloved; for her the expectation and realization of the approaching end of reproductive function mean the abandonment of all hope of fulfilling her natural childbearing destiny. Naturally, therefore, she shows evidence of despair or, instead, a protest reaction. The least susceptible women are those who have had a healthy, happy life, who have made a successful marriage and have raised a healthy family. For them the menopause is merely another milestone in life passed, with every reason to expect the next phase to be no less worth living than those which have gone before.

The psychic symptoms are thus largely conditioned by the circumstances which have evoked them. Irritability and depression are the keynote. The former makes the women short-tempered and intolerant; any slight deviation from the expected course of affairs provokes an exaggerated protest. There may be associated anxiety with vague forebodings of ill health or economic disaster in the future. . Depression may be associated with tearfulness or with sadness, indifference and apathy. The woman may lose all ambition, ceasing to care for her husband, her home and her appearance. As part of the climacteric syndrome changes in libido may occur. There may be an increase as a compensatory reaction to waning reproductive ability, or as a 'last fling' effect; or it may disappear abruptly as in the case, for example, of an unhappily married woman for whom the menopause can serve as an excuse for avoiding distasteful sexual activity. In the well balanced woman, the climacteric need cause no change in libido and many women continue to have normal and satisfying sexual relations long after menstruation has ceased.

The commonest of all climacteric symptoms are the hot flushes which are a manifestation of disturbance of vasomotor control. They may be very occasional and momentary, thereby causing no distress or, at the other extreme, exceedingly frequent and persistent, often accompanied by 
marked sweating and causing great discomfort. Many other symptoms-headaches, joint pains, constipation, urinary disturbances and so onmay occur as part of the syndrome but they are of minor importance as compared with the characteristic ones described above.

Treatment. Successful treatment of the climacteric syndrome requires common sense and delicacy. The routine administration of oestrogen, often for long periods and in excessively high dosage, is strongly to be condemned, just as is the unhelpful nihilistic approach which, taking its stand on the physiological nature of the climacteric, refuses any form of treatment. It is perfectly true that mild cases can be effectively treated by reassurance and perhaps moderate sedation, such as is provided by phenobarbitone gr. $\frac{1}{2}$ B.D. The more severe cases, however, will certainly require oestrogen therapy.

The basic principles of oestrogen therapy for the menopause are as follows: first, oestrogen is never produced continuously under physiological conditions, so that oestrogen treatment should always be in interrupted courses; second, the object of the treatment is to convert an abrupt fall in oestrogen level into a more slowly declining one. It follows, therefore, that if comparatively large doses are given, so that the total oestrogen level is brought up to the previously normal value, symptoms will be certain to reappear every time treatment is stopped. Ignorance of this situation is responsible for most of the so-called ' difficult cases ' who have been on oestrogen for long periods of time, but who relapse miserably each time the oestrogen treatment is stopped.

In general, it is convenient to start treatment with stilboestrol $0.2 \mathrm{mg}$. daily. The treatment should be continued for 28 days and then should be stopped for a fortnight, when it can be resumed at the same dose level. With this dose, most, if not all, of the symptoms will be relieved. Indeed, it is best if an occasional hot flush still occurs while under oestrogen treatment. Generally, some return of symptoms will be experienced during the fortnight off treatment. However, as these monthly courses of treatment proceed, the stage will be reached when symptoms no longer return when treatment is stopped. This is the time to reduce the dose of stilboestrol to $0.1 \mathrm{mg}$. daily. Two or three further interrupted courses of treatment at this level will usually be sufficient to see the end of all significant symptoms. Occasionally, $0.2 \mathrm{mg}$. will prove insufficient to relieve the symptoms adequately; if this is so, the dose may be increased to $0.5 \mathrm{mg}$. daily, but it is seldom necessary to exceed this figure.

In recent years, a vogue has developed for the use of mixed hormone preparations, usually containing ethinyl oestradiol and methyl testo- 3 sterone. The rationale for such treatment is a $\stackrel{\square}{2}$ little dubious, but the general idea is that the two $C$ hormones are synergistic in many of their desirable $\overrightarrow{\vec{F}}$ characteristics, while being mutually antagonistic $\stackrel{5}{?}$ so $f a r$ as some of the undesirable side effects are concerned. By the use of such a mixture, one $\frac{\bar{\sigma}}{\bar{c}}$ is often able to control the climacteric symptoms $\frac{\vec{\sigma}}{\vec{\sigma}}$ with far smaller oestrogen doses than would be $\propto$ possible without the admixture of the androgen. \% Generally speaking, there is little need to ase these $\overrightarrow{0}$ mixed preparations in the average case. It does appear, however, that for the more difficult patient $\vec{\omega}$ who is responding poorly to oestrogen alone, the $\frac{\Omega}{8}$ mixture may be of real value. The general $\frac{0}{3}$ principles mentioned above apply equally to the use of mixed hormone preparations, the starting dose for which would be about two tablets per $\omega$ day.

Where marked psychological aberrations are present, it is obviously necessary to adopt $\vec{\rho}$ psychiatric measures in addition to such hormonal $\frac{}{2}$ therapy as may be required. Since the syndrome $\vec{c}$ is essentially a self-limited one, the prognosis is usually fairly good and it is not often that elaborate psychotherapy is needed.

Except in mild and transient cases, it is a wise rule to subject every patient with climacter menorrhagia to a diagnostic dilatation and curettage This procedure appears to be curative in a fair proportion of patients; estimates vary between $30 \%$ and 60 per cent. If the curettage fails to reveal $\stackrel{\varnothing}{\propto}$ any endometrial pathology, but the menorrhagia $\vec{\overrightarrow{ }}$ nevertheless persists, the choice of medical or $\frac{3}{3}$ surgical treatment has then to be made. The surgical alternatives are a radium menopause or hysterectomy. The advantages of the former are its simplicity and freedom from immediate compli- 3 . cations. The disadvantages of a radium menopause are: that it frequently precipitates a severe climacteric syndrome, with marked genital $\delta$ atrophy and requiring medical treatment; the $₹$ possibility that a carcinoma of the body of the 을 uterus may have been overlooked; or that there $\rightarrow$ may be an increased risk of uterine malignancy after irradiation. Should surgery later become $\frac{N}{N}$ necessary, previous irradiation may have caused 0 scarring and so render it very difficult. The chief $\mathbb{N}$ advantage of hysterectomy is that it obviates these $\underset{\omega}{N}$ various difficulties; its disadvantage is the risk of $\sigma^{\circ}$ complications inherent in all surgery, together 0 with the fear which the idea of a surgical operation $\mathbb{\mathscr { C }}$ engenders in many patients.

The above considerations suggest that medical 0 treatment should ordinarily be given a trial before surgery is used. For regular menorrhagia the $\stackrel{\mathbb{Q}}{\overparen{D}}$ patient may be instructed to take ethisterone $\stackrel{\mathbb{Q}}{\mathscr{Q}}$ 
I $5 \mathrm{mg}$. and methyl testosterone* $5 \mathrm{mg}$. B.D. sub-lingually, commencing about a week before the period is expected and continuing until the third day. This treatment will often restore the heavy period to more normal proportions (Swyer, 1953). When the bleeding is prolonged and irregular, the choice lies mainly between treatment with progesterone to produce 'medical curettages,' or with oestrogens.

A daily intramuscular injection of progesterone $25 \mathrm{mg}$. and testosterone $50 \mathrm{mg}$. for four days will usually terminate a prolonged bout of bleeding. A few days later a self-limited progesterone withdrawal bleeding will occur. Repetition of the progesterone injections (without the testosterone) at approximately monthly intervals will usually produce regular normal 'periods.'

Oestrogen therapy depends upon the fact that a sufficient dose of oestrogen will usually terminate a bout of bleeding. A convenient dose is commonly stilboestrol $2 \mathrm{mg}$., or ethinyl oestradiol o.I mg. daily. This should be continued for a total of twenty days. If there has been no noticeable effect on the extent of bleeding within forty-eight hours, the dose should be doubled and continued at this higher level for the remainder of the twenty days. Within a week or ten days after stopping the oestrogen treatment, a withdrawal bleeding will occur and on the fifth day another course of oestrogen therapy should be begun. In this way the irregular bleeding is converted into

* Ethisterone and methyl testosterone, in the above dosage, is available in a combined tablet as Androgeston (British Schering). regular cycles and the dose can gradually be reduced in succeeding months. Eventually, a stage will be reached where the dose will be too small to provoke an oestrogen withdrawal bleeding and the menopause will have been established. If, during the course of oestrogen administration, the bleeding stops but begins again before the course is completed, further oestrogen should be withheld for five days and then a new course commenced as before (Swyer, 1950).

Fears have been entertained that administration of oestrogens to women at the menopause may lead to carcinogenesis. There is no evidence whatever to substantiate such a fear, and, in any case, provided the oestrogen is given discontinuously as recommended above, the likelihood, even on theoretical grounds, of its exerting a carcinogenic action is extremely remote.

Failure to respond to hormonal treatment is not uncommon; it is obviously unwise to persist in such treatment if it is proving ineffective and recourse should then be had to surgical measures without undue delay.

\section{BIBLIOGRAPHY}

HAMBLEN, E. C. (1947), 'Endocrinology of Women,' Charles C. Thomas, Springfield, Illinois.

Lancet (1933), 'An Investigation of the Menopause in One Thousand Women,' $i$, ro6.

MANDL, A. M., and ZUCKERMAN, S. (I95 Ia), f. Endocrinol, 7, 103.

MANDL, A. M., and ZUCKERMAN, S. (195 Ib), Ibid., 7, I 12.

MANDL, A. M., and ZUCKERMAN, S. (195Ic), Ibid., 7, 190.

MANDL, A. M., ZUCKERMAN, S., and PATTERSON, H. D. (1952), Ibid., 8, 347.

SIMPKINS, C. S. (1932), Amer. F. Anat., 51, 465.

SWEZY, O., and EVANS, H. M. (1930), F. Morphol. Physiol, 49, 543 .

SWYER, G. I. M. (1950), Brit. Med. F., I, 626.

SWYER, G. I. M. (1953), Lancet ii, 347.

Copies of Title Page and Index for Vol. 29 of The Postgraduate Medical Journal are available on request. See under for binding particulars.

\section{NOTICE OF SPECIAL INTEREST TO SUBSCRIBERS:}

\author{
"WHY NOT HAVE YOUR COPIES OF THIS \\ JOURNAL BOUND INTO YEARLY VOLUMES?"
}

\begin{abstract}
Arrangements have now been made to have the twelve monthly issues fully bound in dark green pin head cloth, lettered in gilt on spine with name of Journal, Volume Number and year, complete with index at front, 17s. 6d. per Volume, post free. A limited number of out of print journals are available to bind into volumes and make your library complete. Price on application giving details of issues required to complete back volumes.
\end{abstract}

